\title{
D isección aórtica y síndrome de Marfán en el embarazo. A propósito de un caso clínico
}

\author{
Christian Espinoza $\mathbf{S}^{\mathbf{1}}$, Rafael Selman $\mathbf{A}^{\mathbf{1}}$, Felipe Pauchard $\mathrm{T}^{\mathrm{a}}$, \\ Juan Rivera $F^{1}$, Sebastián Iturra U ${ }^{1}$, Fernando Montecinos $\mathbf{R}^{2}$, \\ Héctor Eliash $D^{2}$, Francisco Undurraga $\mathrm{H}^{\mathrm{a}}$.
}

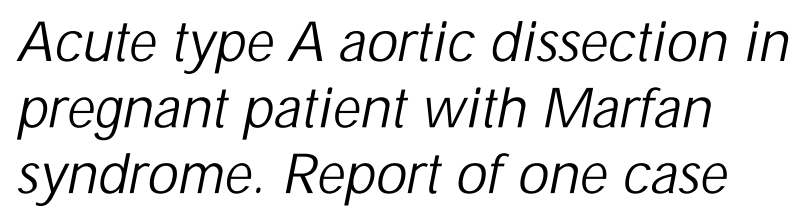

Aortic dissection is a life-threatening disease that requires immediate surgical intervention. Marfan syndrome is a hereditary disease with an autosomal dominant transmission, which affects the connective tissue, with skeletal, cardiovascular and ocular involvement. It is one of the most prevalent connective tissue disorders, presenting a risk of aortic dissection of approximately $1 \%$ even without dilatation of the aorta. When dissection occurs during pregnancy and requires surgical intervention (type A dissection), maternal mortality is high (20\%-30\%). We report a 38 year-old woman with Marfan syndrome that had an acute type A aortic dissection and severe aortic regurgitation at 37 weeks of gestation. The patient underwent a cesarean section and delivered a healthy baby. Afterwards, aortic valve repair and ascending aortic replacement was successfully performed under circulatory arrest with deep hypothermia. Additionally mitral valve repair for degenerative disease with posterior, autologous pericardium mitral valve ring was performed (Rev Méd Chile 2009; 137: 98-100).

(Key words: Aortic aneurysm; Marfan syndrome; Pregnancy complications, cardiovascular)

Recibido el 7 de noviembre, 2007. Aceptado el 28 de julio, 2008.

${ }^{1}$ Servicio Cardiovascular, Instituto Nacional del Tórax. ${ }^{2}$ Servicio Ginecología y Obstetricia,

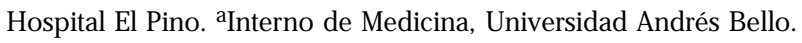

$\mathrm{E}^{1}$ síndrome de Marfán es una enfermedad hereditaria, de transmisión autosómica dominante, que afecta al tejido conectivo, con manifestaciones esqueléticas, oculares y cardiovasculares. Corresponde a uno de los trastornos del tejido conectivo más prevalentes, con una incidencia de 1 en 10.000 a 20.000 personas $^{1}$. Presentan un

Correspondencia a: Dr. Christian Espinoza Silva. Servicio Cardiovascular, Instituto Nacional del Tórax. Infante 717, Providencia, Santiago.

E mail: cardiocirugia@clinicatabancura.cl riesgo de disección aórtica, incluso sin dilatación de la aorta, de alrededor de $1 \% 2,3$. Cuando se produce durante el embarazo y requiere intervención quirúrgica (disección tipo A), la mortalidad materna es elevada (20\% a $30 \%)^{4}$.

Por otra parte, el embarazo genera una serie de cambios fisiológicos a nivel del sistema cardiovascular, debido a las modificaciones hormonales propias de la gestación, como son un incremento progresivo del gasto cardíaco, el cual puede aumentar hasta $30 \%$ por encima de los valores preembarazo, una caída de las resistencias vascu- 
lares sistémicas, en gran parte relacionado con el desarrollo de un lecho de baja resistencia como es la placenta y una anemia relativa, por hipervolemia, todo lo cual produce una menor tolerancia de las cardiopatías e incluso puede conducir al desarrollo de complicaciones tanto a nivel materno, como fetal o neonatal.

\section{CASO CLÍNICO}

Mujer de 38 años, con antecedentes de tres embarazos y dos partos normales, con diagnóstico ecocardiográfico de síndrome de Marfán hecho en el año 2000, antecedente de enfermedad reumática con compromiso mitral y dos hermanos fallecidos de disección aórtica por enfermedad de Marfán. Presentaba un embarazo de 37 semanas, con buen control gestacional, sin complicaciones, hasta que, sin un desencadenante específico, presentó un intenso dolor retroestemal transfixiante, acompañado de signos vegetativos, por lo que consultó en el Servicio de Urgencia de Ginecología y Obstetricia del Hospital El Pino, en donde se comprobó la vitalidad fetal y rápidamente fue vista por el equipo médico-quirúrgico, que demostró una disección aórtica tipo A, por ecocardiografía transtorácica bidimensional y fue derivada al Instituto Nacional del Tórax.

Se coordinó a los equipos obstétricos del Hospital El Pino y Cardioquirúrgico y Anestésico del Instituto Nacional del Tórax, realizando una doble intervención sucesiva. Previo a la cirugía se realizó ecocardiograma bidimensional que mostró un flap de disección aórtica ascendente, que comprometía desde $2,8 \mathrm{~cm}$ del anillo valvular, con insuficiencia aórtica moderada, insuficiencia mitral severa y derrame pericárdico leve. Función ventricular adecuada.

En primer lugar se abordó la parte obstétrica, para extraer al feto y evitar el daño neonatal, realizando una cesárea de urgencia más una histerectomía subtotal y anexectomía izquierda. El recién nacido presentó una depresión respiratoria de corta duración, asociada probablemente a la parte anestésica, la que no determinó secuelas posteriores, con una estadía habitual en el Servicio de Neonatología del Hospital el Pino.

En una segunda etapa, inmediatamente posterior a la cesárea, se colocó a la paciente en circulación extracorpórea (CEC) con canulación por arteria subclavia derecha y venosa bicava estándar. Se constató la presencia de un hematoma disecante en la posición habitual, sin derrame pericárdico significativo. Durante la CEC se inició el enfriamiento, se realizó la comección valvular aórtica con resuspensión del aparato valvular. Posteriormente, se realizó un paro circulatorio a $20 \mathrm{C}^{\circ}$ con circulación cerebral anterógrada. Se exploró el falso lumen aórtico demostrándose permeabilidad de éste, evidenciando el sitio de rotura intimal a dos centímetros del plano valvular. Se realizó el reemplazo de la aorta ascendente, con una prótesis de dacrón microporosa precoagulada de $26 \mathrm{~mm}$

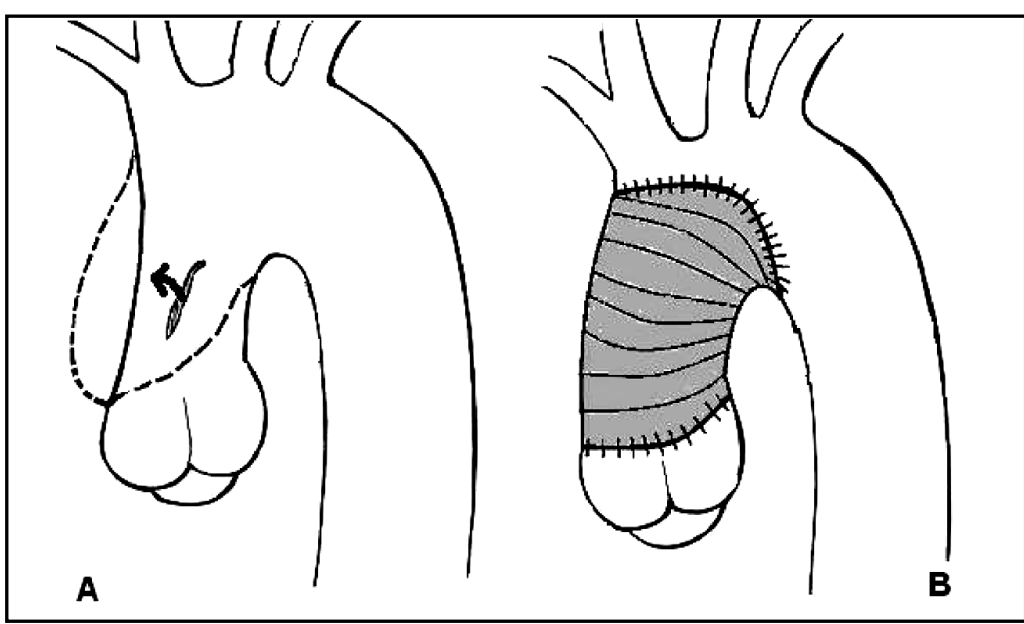

Figura 1. Visión esquemática de la cirugía sobre la aorta ascendente en una disección tipo A. A: Imagen de la disección aórtica que afecta la aorta ascendente, con un sitio de ruptura intimal a dos centímetros sobre el plano valvular (flecha). B: Reemplazo de la aorta ascendente por una prótesis tubular de dacrón. 
(Figura 1). Finalizado esto se realizó la anastomosis proximal y el recalentamiento, durante este peńodo se realizó la plastia mitral con anillo de pericardio autólogo. El tiempo total de paro circulatorio con perfusión cerebral anterógrada selectiva fue de 23 min, el clampeo aórtico de 138 min y la CEC de 187 min el tiempo de reperfusión fue de $45 \mathrm{~min}$.

La paciente requirió 5 días de hospitalización en UCI con apoyo inotrópico, estando las primeras 10 $\mathrm{h}$ en ventilación mecánica invasiva. Posteriormente evolucionó con cuadros febriles por lo que se dejó antibióticos empíricos de amplio espectro (cultivos negativos) y fue dada de alta sin actividad séptica y en buen estado general a los 40 días. Los controles alejados no han revelado ninguna complicación posterior. Cuatro meses post cirugía se encontraba bien, con capacidad funcional grado II, ortopnea de 2 almohadas y no había presentado edema. Su hijo presenta una evolución favorable, sin estigmas de enfermedad de Marfán y se mantiene en control en cardiología.

\section{Comentarios}

Las pacientes con síndrome de Marfán que se embarazan pueden sufrir complicaciones en cualquier momento del embarazo o en el período postparto, sin embargo la mayoría se presentan durante el segundo y tercer trimestre de éste ${ }^{5}$. Las complicaciones cardiovasculares más comunes son el aumento progresivo del diámetro de la raíz

\section{REFERENCIAS}

1 Ramírez F, Godfrey M, Lee B, Tsipouras P. Marfan syndrome and related disorders. En: The Metabolic and Molecular Basis of Inherited Disease, $7^{\text {th }}$ ed, Scriver, CR, Beaudet, AL, Sly, WS, et al (Eds), McGraw Hill, New York 1995; 4079.

2. Rossiter JP, Repke, JT, Morales AJ, Murphy EA, Pyeritz RE. A prospective longitudinal evaluation of pregnancy in the Marfan syndrome. Am J Obstet Gynecol 1995; 173: 1599-606.

3 Lipscomb KJ, Smith JC, Clarke B, Donnai P, Harris R. Outcome of pregnancy in women with Marfan's syndrome. Br J Obstet Gynaecol 1997; 104: 201-6.

4. Pomini F, Mercoglano D, Cavaluetti C, Caruso A, aórtica, iniciándose en los senos de Valsalva, aneurisma aórtico ascendente que puede disecarse, ruptura aórtica, regurgitación aórtica o las tres en forma simultánea 6 . Los factores de riesgo más comunes, para desarrollar una disección tipo A en pacientes que sufren de síndrome de Marfán, son el aumento del diámetro de la raíz aórtica sobre $40 \mathrm{~mm}$ o un aumento del tamaño aórtico durante el embarazo ${ }^{2}$.

La disección aórtica es una emergencia quirúrgica, con mortalidad que clásicamente bordea $1 \%$ por cada hora transcurrida, por lo que requiere de reparación quirúrgica inmediata. Existen muy pocos reportes de casos en que coincidan el síndrome de Marfán, embarazo y disección aórtica. Las mujeres embarazadas con disección aórtica tipo A tienen indicación de cirugía de inmediato, a pesar de los probables riesgos fetales ${ }^{7}$.

La cirugía del arco aórtico tiene indicación de paro circulatorio con hipotermia profunda y perfusión cerebral anterógrada. El solo uso del bypass cardiopulmonar, por cualquier motivo, puede llegar a tener una mortalidad materna de 3\%. En relación al feto, la mortalidad puede llegar a $20 \%$, lo que se explica por la hipotermia, la que reduce el flujo placentario y aumenta las contracciones uterinas ${ }^{4}$, todo lo cual, se ve agravado al utilizar hipotermia profunda y paro circulatorio. En definitiva la coincidencia de síndrome de Marfán con embarazo y disección aórtica tiene muy baja frecuencia y es un desafío clínico sin conceptos terapéuticos definidos ${ }^{8}$.

Pomini P. Cardiopulmonary bypass in pregnancy. Ann Thorac Surg 1996; 61: 259-68.

5. Elkayam, U, Ostrzega, E, Shotan A, Mehra A. Cardiovascular problems in pregnant women with the Marfan syndrome. Ann Intern Med 1995; 123: 117-22.

6. Dianna M Milewicz, Harry C Dietz, D Craig Milier. Treatment of Aortic Disease in Patients With Marfan Syndrome. Circulation 2005; 111; e150-e157.

7. IMMER FF, BANSI AG, IMmER-BANSI AS, MCDOUgall, ZeHR KJ, SchafF HV ET AL. Aortic dissection in pregnancy: analysis of risk factors and outcome. Ann Thorac Surg 2003; 76: 309-14.

8. Seeburger J, Mohr FW, Falk V. Acute Type A Dissection at 17 Weeks of Gestation in a Marfan Patient. Ann Thorac Surg 2007; 83: 674-6. 